\title{
CANONICAL FUNCTIONS OF ASYMPTOTIC DIFFRACTION THEORY ASSOCIATED WITH SYMPLECTIC SINGULARITIES
}

\author{
ANDRZEJ HANYGA \\ Institute for Solid Earth Physics, University of Bergen \\ Allégaten 41, N-5007 Bergen, Norway \\ E-mail: andrzej@ifjf.uib.no
}

\begin{abstract}
A general method of deriving canonical functions for ray field singularities involving caustics, shadow boundaries and their intersections is presented. It is shown that many time-domain canonical functions can be expressed in terms of elementary functions and elliptic integrals.
\end{abstract}

1. Introduction. The subject of this paper is a systematic method for constructing local asymptotic expansions of wave fields at arbitrary ray field singularities (caustics, shadow boundaries, critical rays etc.). The method is based on lifting the ray field or its singularity to the cotangent bundle and deriving an asymptotic expansion in terms of oscillatory integrals from a local representation of the resulting manifold in terms of a generating family of functions. Existence of generating families of functions is generally established by symplectic reduction $[25,16]$. The scope of this approach exceeds the scope of Maslov theory since it is not limited to caustic singularities. In contradistinction to the Maslov theory, the time evolution of the field at a point $x$ is expressed in terms of the values of the eikonal function and ray amplitudes at $x$ only. The asymptotic series of oscillatory integrals thus obtained satisfies the PDEs provided the eikonal function and the ray amplitudes satisfy the eikonal equation and the transport equations.

In a neighbourhood of a symplectic singularity the asymptotic expansion is transformed to an appropriate canonical form. The result is a uniformly asymptotic series of canonical time-domain functions and their indefinite integrals. The arguments of the canonical functions can be expressed in terms of eikonals while their linear coefficients are linear functions of ray amplitudes.

We emphasize practical benefits of using time-domain canonical functions in numerical applications instead of the more familiar frequency-domain solutions. In particular we

1991 Mathematics Subject Classification: Primary 41A60; Secondary $53 Z 09$.

The paper is in final form and no version of it will be published elsewhere. 
list the cases in which explicit analytic solutions are available in the time domain. The literature on time-domain canonical functions is scarce. Time-domain canonical functions for simple caustics were derived in $[4,24,3,15]$. The canonical expressions given in [4] and in [3] are non-uniform while the expression derived in [24] does not satisfy the radiation condition. The method presented in this paper is an extension of [15]. A frequencydomain theory corresponding to Sections $2-4$ for $\mathcal{C}^{\infty}$ functions and manifolds is discussed at length in [6].

Two kinds of asymptotic solutions of PDEs are considered in this paper:

(i) "high-frequency" asymptotic expansions of solutions $U(x, \lambda)$ of

$$
P\left(x, D_{x},-\mathrm{i} \lambda\right) U(x, \lambda)=0
$$

for $\lambda \rightarrow \infty$,

(ii) wavefront expansions of solutions $V(x, t)$ of

$$
P\left(x, D_{x}, D_{t}\right) V(x, t)=0
$$

where $P$ is a PDE or a pseudo-differential operator, $x \in \mathcal{X}$ and $\mathcal{X}$ is a domain in $\mathbb{R}^{n}$.

Both kinds of expansions are constructed in terms of an eikonal function $S(x)$ satisfying the Hamilton-Jacobi equation

$$
H(x, p)=0
$$

with

$$
p=\mathrm{d} S(x)
$$

and

$$
H(x, p)=\operatorname{det} P_{0}(x, p)
$$

where $P_{0}$ denotes the principal symbol of $P\left(x, D_{x}, D_{t}\right)$. The eikonal equation can be solved by integrating the bicharacteristic equations

$$
\frac{\mathrm{d} x}{\mathrm{~d} \tau}=\frac{\partial H}{\partial p}, \quad \frac{\mathrm{d} p}{\mathrm{~d} \tau}=-\frac{\partial H}{\partial x}
$$

with appropriate initial data

$$
x(0, u)=x^{(0)}(u), p(0, u)=p^{(0)}(u), \quad u \in \mathcal{U}
$$

satisfying $H\left(x^{(0)}, p^{(0)}\right)=0$, related to an initial eikonal $S^{(0)}$ by the equation

$$
p_{i}^{(0)}(u) \mathrm{d} x_{i}^{(0)}(u)=\mathrm{d} S^{(0)}(u) \quad \forall u \in \mathcal{U}
$$

and such that the Hamiltonian vector field $X_{H}$ of $H$ is nowhere tangent to $\left(x^{(0)}(u), p^{(0)}(u)\right)$. The eikonal $S(x)$ is then obtained by integrating along the rays $x(\cdot, u)$

$$
\frac{\mathrm{d} \check{S}}{\mathrm{~d} \tau}=p_{i} \frac{\partial H}{\partial p_{i}}
$$

with the initial condition $\check{S}(0, u)=S^{(0)}(u)$ and changing the coordinates from $(\tau, u)$ to $x$ if possible.

The solutions of equations (3)-(4) are multi-valued. In order to overcome this difficulty the problem is reformulated as follows. Instead of solving Eqs. (3)-(4) for the eikonal function $S$ we look for a Lagrangian immersion $\iota: \Omega \rightarrow \Lambda \subset T^{*} \mathcal{X}, \Omega \subset \mathbb{R} \times \mathcal{U}$, satisfying 
$\mathcal{I} \subset \Lambda \subset H^{-1}(0)$, where $\mathcal{I}$ denotes the image of the isotropic immersion $\mathcal{U} \ni u \rightarrow$ $\left(x^{(0)}(u), p^{(0)}(u)\right)$, cf. [2, 9]. If we assume that the vector field $X_{H}$ is nowhere tangent to $\mathcal{I}$, the Lagrangian immersion $\iota$ and the function $\breve{S}$ can be constructed by integrating equations (5) with the initial data (6) [9], but the coordinate change from $(\tau, u)$ to $x$ is no longer required.

Geometrical Theory of Diffraction (GTD) [18] leads to more complicated geometrical constructions. In order to satisfy the boundary conditions at boundaries and interfaces (discontinuity surfaces of the coefficients of the differential operator $P$ ) reflected, transmitted and diffracted ray fields have to be introduced. An interface or a diffracting edge $x=g(v), v \in \mathbb{R}^{\nu}, \nu=2$ or 1 , acts as a secondary source of a ray field satisfying the initial condition

$$
x(0, v)=g(v), p_{i}(0, v) \mathrm{d} g^{i}(v)=p_{i}^{(I)}(v) \mathrm{d} g^{i}(v)
$$

where $p^{(I)}=\mathrm{d} S^{(I)}$ and $S^{(I)}(x)$ denotes the eikonal of the incident field, as well as equation (3) with an appropriate Hamiltonian $H$. The eikonal is then determined from equation (8) with the initial conditions

$$
S(g(v))=S^{(I)}(g(v)) .
$$

In particular, an interface breaking off at an edge produces a reflected and an edgediffracted ray field generated by the secondary sources on the reflecting surface and at the edge. The associated Lagrangian submanifolds are denoted by $\Lambda_{P}$ and $\Lambda_{D}$. The shadow boundary $\mathcal{B}_{P}$ for reflected rays is then spanned by those reflected rays which coincide with edge-diffracted rays. The associated reflected and edge-diffracted bicharacteristics also coincide since the initial conditions at the edge and the Hamiltonians for both kinds of rays are identical. Consequently the shadow boundary $\mathcal{B}_{P}$ is the projection of

$$
\mathcal{I}_{P}=\Lambda_{P} \cap \Lambda_{D} \subset T^{*} \mathcal{X}
$$

on $\mathcal{X}$. The reflected rays correspond to the points of $\Lambda_{P}$ on just one side of the intersection but uniform asymptotic expansions presented in Sections $5-6$ require an analytic continuation of the Lagrangian submanifold beyond the intersection. The same remark applies to the shadow boundaries of direct and transmitted rays, with appropriate diffracted rays.

Tangential or critical incidence of rays on a boundary or an interface is another source of diffracted rays, cf. [19]. The methods described below apply to the first of these cases. We shall however limit our considerations to edge and vertex diffraction.

The method developed below depends on the possibility of computing evanescent wavefields in caustic shadows by tracing complex rays. This in turn implies that the Lagrangian submanifold should be a complex analytic variety in the neighbourhood of its caustic singularities. Consequently, we shall assume that both $\mathcal{X}$ and $\Omega$ is a domain in $\mathbb{C}^{n}$ and all the mappings are holomorphic.

Notation. The following symbols are used in the paper:

$$
\begin{aligned}
\langle v \mid w\rangle & \text { - scalar product in } \mathbb{R}^{3} \\
\langle v|A| w\rangle & =\langle v \mid A w\rangle \\
\langle f, g, \ldots\rangle & - \text { an ideal spanned by } f, g, \ldots
\end{aligned}
$$


2. Singularities of ray fields and oscillatory integrals. Broadly speaking, a ray field singularity is a locus of points at which the amplitudes $A_{k}(x)$ of the primitive asymptotic ray expansions

$$
U \sim \sum_{k=0}^{\infty}(\mathrm{i} \lambda)^{-k} A_{k}(x) \exp [\mathrm{i} \lambda S(x)]
$$

or

$$
V \sim \sum_{k=0}^{\infty} A_{k}(x) f_{k}(t-S(x))
$$

with

$$
f_{k+1}^{\prime}(\tau)=f_{k}(\tau)
$$

tend to infinity. It will be shown later that ray field singularities are either projections on $\mathcal{X}$ of the intersections of different branches of the Lagrangian manifolds over $\mathcal{X}$ (more specifically self-intersections and folds) or projections of the singularities of the Lagrangian manifolds. In particular, caustics are projections of the folds $\mathcal{F}$ of $\Lambda$ over $\mathcal{X}$, i.e. singularities of the projection $\pi: T^{*} \mathcal{X} \rightarrow \mathcal{X}$ restricted to $\Lambda$ :

$$
\mathcal{F}=\{q \in \Lambda \mid \operatorname{corank} \mathrm{D} \pi(q)>0\} .
$$

At the projections of the self-intersections $\mathcal{I}$ of $\Lambda$ the amplitudes of diffracted rays are infinite. A related phenomenon is the blow-up of head-wave amplitudes along the critically reflected rays, which are associated with the coalescence of reflected and head-wave rays.

We now show how an asymptotic solution of equation (1) can be constructed in terms of oscillatory integral expansions.

TheOREM 1 [25]. A Lagrangian submanifold $\Lambda$ can locally be expressed by the formula

$$
\Lambda=\left\{(x, p) \in T^{*} \mathcal{X} \mid \exists u \in \mathcal{U}: p=\frac{\partial \phi}{\partial x}, \frac{\partial \phi}{\partial u}=0\right\}
$$

where $\phi: \mathcal{U} \times \mathcal{X} \rightarrow \mathbb{C}, \mathcal{U} \subset \mathbb{C}^{m}$, is analytic and satisfies the condition

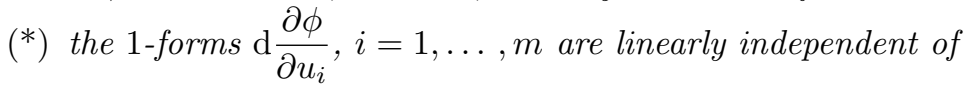

$$
\mathcal{M}=\left\{(u, x) \in \mathcal{U} \times \mathcal{X} \mid \frac{\partial \phi}{\partial u}=0\right\} .
$$

Condition $\left(^{*}\right)$ excludes Lagrangian manifolds with singularities, which appear in smooth body diffraction.

Let $\operatorname{pr}_{\mathcal{X}}: \mathcal{U} \times \mathcal{X} \rightarrow \mathcal{X}$ denote the projection onto $\mathcal{X}$. If $\operatorname{det} \partial^{2} \phi / \partial u^{2} \neq 0$ at some $(u, x) \in \mathcal{M}$ then $\operatorname{pr}_{\mathcal{X}} \mid \mathcal{M}$ is locally a submersion. In this case $\pi \mid \Lambda$ is a submersion in a neighbourhood of $p=\frac{\partial \phi}{\partial x}(u, x)$. Consequently

$$
\mathcal{F}=\left\{(x, p) \in \Lambda \mid \exists u \in \mathcal{U}: p=\frac{\partial \phi}{\partial x}, \frac{\partial \phi}{\partial u}=0, \operatorname{det} \frac{\partial^{2} \phi}{\partial u^{2}}=0\right\}
$$

Suppose now that $\Lambda$ has been constructed as described in Section 1 and $\iota$ denotes the immersion $\mathcal{M} \rightarrow \mathcal{U} \times \mathcal{X}$. If $\Omega$ is a connected open subset of $\mathcal{M}$ such that $\operatorname{det} \partial^{2} \phi / \partial u^{2} \neq 0$ on $\Omega$, then a comparison of equations (4) and (10) shows that $\left(\operatorname{pr}_{\mathcal{X}} \mid \mathcal{M}\right)_{*}^{-1} \iota_{*} \phi$ differs from 
the eikonal by a constant. This suggests that the asymptotic solution of equation (1) for $\lambda \rightarrow \infty$ can be written in the form of a compound asymptotic [9]

$$
U(x, \lambda) \sim \int_{\mathcal{U}} a(u, x, \lambda) \exp [\mathrm{i} \lambda \phi(u, x)] \mathrm{d} u
$$

where

$$
a(u, x, \lambda) \sim \sum_{k=0}^{\infty}(\mathrm{i} \lambda)^{-k} a_{k}(x, u) .
$$

For comparison with ray expansions, the stationary phase method [7, 9] can be applied to expression (12). The eikonals $S^{(j)}(x)$ and ray amplitudes $A^{(j)}(x)$ are obtained by substituting the solutions $u=u^{(j)}(x)$ of $\partial \phi / \partial u=0$ in $\phi$ and in

$$
(2 \pi /|\lambda|)^{-m / 2} \exp \left(\mathrm{i} \pi \operatorname{sgn} \phi_{u u} / 4\right) a_{0}(x, u) /\left|\operatorname{det} \phi_{u u}\right|^{1 / 2}
$$

respectively, where $\phi_{u u}:=\partial^{2} \phi / \partial u^{2}$. The following theorem [15] describes the necessary conditions for expression (12) to be an asymptotic solution of equation (1):

THEOREM 2. If $U(x, \lambda)$ satisfies equation (1) in the asymptotic sense:

$$
P\left(x, D_{x}, \lambda\right) U(x, \lambda)=\mathrm{O}\left[\lambda^{-\infty}\right]
$$

then

(i) the Lagrangian submanifold

$$
\Lambda:=\left\{(x, p) \mid \frac{\partial \phi}{\partial u}=0, \frac{\partial \phi}{\partial x}=p\right\}
$$

satisfies the generalized eikonal equation $\Lambda \subset\left\{(x, p) \mid \operatorname{det} P_{0}(x, p)=0\right\}$;

(ii) the function $a_{0}(u, x)$ satisfies the polarization equations:

$$
a_{0}(x)=c(x) r\left(x, \frac{\partial \phi}{\partial x}\right)+\mathrm{O}\left[\frac{\partial \phi}{\partial u}\right]
$$

where $r(x, p)$ is a unit vector in $\operatorname{ker} P_{0}(x, p)$ for $(x, p) \in H^{-1}(0)$;

(iii) the half-form $\rho:=c\left(\mathrm{~d} x^{1} \wedge \ldots \mathrm{d} x^{n}\right)^{1 / 2}$ [9] satisfies the transport equation

$$
\mathcal{L}_{X_{H}} \log \rho+\left\langle r\left|\mathrm{i} P_{1}-\frac{1}{2} \frac{\partial^{2} P_{0}}{\partial p_{j} \partial x^{j}}\right| r\right\rangle=0
$$

where $X_{H}=\frac{\partial H}{\partial p_{i}} \frac{\partial}{\partial x^{i}}-\frac{\partial H}{\partial x^{i}} \frac{\partial}{\partial p_{i}}$ is the Hamiltonian vector field associated with $H$.

Eq. (\#) is a generalization of the scalar transport equations derived in $[6,9]$. An asymptotic solution of equation (2) can be constructed as follows:

$$
V(x, t)=(1 / 2 \pi) \int_{-\infty}^{\infty} \frac{|\lambda|^{m / 2}}{(2 \pi)^{m / 2+1}} \exp \left(-\mathrm{i} m \frac{\pi}{4} \operatorname{sgn} \lambda\right) U(x, \lambda) \exp (-\mathrm{i} \lambda t) \mathrm{d} \lambda .
$$

The additional frequency filter in the integrand of (13) ensures that the lowest-order contribution of a non-degenerate minimum of $\phi(\cdot, x)$ to $V(x, t)$ has the form of a Dirac delta $A(x) \delta(t-T(x))$. Substituting equation (12) in equation (13) and carrying out integration over $\lambda$ one obtains the following integral representation of $V(x, t)$ for $m=1$ 
and $m=2$, respectively:

$$
V(x, t) \sim \sum_{l=0}^{\infty} F_{l}\left(t, x, a_{l}\right)
$$

where

$$
\frac{\partial F_{l+1}(t, x ; a)}{\partial t}=F_{l}(t, x, a), l=0, \ldots
$$

and

$$
F_{0}(t, x ; a)=1 /(\sqrt{2} \pi) \frac{\partial}{\partial t} \int_{\phi<t} a(u, x)[t-\phi(u, x)]^{-1 / 2} \mathrm{~d} u
$$

for $m=1$ and

$$
F_{0}(t, x, a)=\frac{1}{2 \pi} \frac{\partial}{\partial t} \int_{\mathcal{U}} a(u, x) \delta(t-\phi(u, x)) \mathrm{d} u
$$

for $m=2$.

Numerical applications of Eqs. (16) and (17) require computation of the phase function $\phi$. This is feasible in some cases, e.g. in the case of caustic singularities [20].

In order to account for evanescent fields in caustic shadows it is necessary to introduce complex rays joining the source to the points in the shadow. This requirement is met by assuming that the phase and amplitude function are analytic in some neighbourhood of every caustic. In this case the Lagrangian submanifold is an analytic variety and the caustics appear as the projection of its locus of singular points [11].

3. Reduction of general oscillatory integrals to canonical forms. We shall apply singularity theory $[1,22]$ to transform the phase function $\phi(u, x)$ to a standard polynomial $\psi(w, y)$ with respect to the integration variables $w$, with some $x$-dependent coefficients $y(x)$. The specific polynomial is related to the singularity at hand.

The transformations are local and consequently a partition of unity $\left\{e_{k}\right\}$ has to be applied to separate the contributions of different unrelated "organizing" singularities as well as the contributions of non-singular subdomains of $\mathcal{U}$, for a given point $x \in \mathcal{X}$ and a neighbourhood of it. The Jacobians of all the local transformations described below are absorbed in the amplitude.

Consider a neighbourhood of a point $\left(0, x_{0}\right) \in \mathcal{M}$ such that $\rho:=\operatorname{corank} \phi_{u u}>0$. By the parametric version of the Splitting Lemma [22] the phase function $\phi$ can be locally transformed to the form

$$
\tilde{\phi}(v, w, y)=\sum_{l=\rho+1}^{m} \frac{1}{2} \sigma_{l} v_{l}^{2}+\psi(w, y)
$$

where $w \in \mathbb{C}^{\rho}, \sigma_{l} \in\{1,-1\}$ and $\operatorname{rank} \psi_{w w}=0$, by a transformation $u \rightarrow(v, w)=f(u, x)$, $y=F(x)$. The "inessential" variables $v$ are eliminated by applying the stationary phase method to the integral over $v$. This leaves a factor $N=(2 \pi / \lambda)^{(m-\rho) / 2} \exp \left[i \pi \sum_{l=1}^{\rho} \sigma_{l} / 4\right]$, which is irrelevant for the following derivations. The function $\psi$ is chosen to be a universal unfolding of a singularity in the Thom-Arnold list $[1,22]$. The asymptotic contribution 
of the singularity to the wave field $U(x, t)$ assumes the following form

$$
\tilde{U} \sim N \int_{\mathcal{W}} \mathrm{d} w b(w, x, \lambda) e^{\mathrm{i} \lambda \psi(w, y(x))}
$$

where $\mathcal{W} \subset \mathbb{R}^{\rho}$ and $b=a J e_{k}, J=\partial(u) / \partial(v, w)$ and we note that $b \mid \partial \mathcal{W}=0$.

We then apply the generalized Malgrange preparation theorem to obtain an expansion of the amplitude modulo $\psi_{w}$ :

$$
b=P(w, x)+\sum_{l=1}^{\mu} f_{l}(w, x) \frac{\partial \psi}{\partial w_{l}}
$$

where $P(w, x)$ is a minimal polynomial in $w$ satisfying (19) with coefficients which are holomorphic functions of $x$ and $f_{l}$ are some holomorphic functions. Integrating by parts and noting that $b \mid \partial \mathcal{W}=0$ we find that the contribution of the second term on the righthand side is $\mathrm{O}[1 /(\mathrm{i} \lambda)]$. The $\mathrm{O}[1 /(\mathrm{i} \lambda)]$ correction is then accounted for by rearranging the asymptotic expansion.

For each monomial $c_{\alpha} w^{\alpha}$ in $P$ there is a corresponding monomial $y_{\alpha} w^{\alpha}$ in $\psi$. Consequently

$$
\tilde{U} \sim \frac{N}{\mathrm{i} \lambda} \sum_{\alpha} c_{\alpha} \frac{\partial}{\partial y_{\alpha}} \int_{\mathcal{W}} \mathrm{e}^{\mathrm{i} \lambda \psi} \mathrm{d} w+\text { h.o.t. }
$$

The asymptotic contribution of the singularity to the wave field $V(x, t)$ is obtained by the argument of Section 2 :

$$
F(t, x) \sim \sum_{r=0}^{\infty} \sum_{\alpha} c_{r \alpha}(x) \frac{\partial}{\partial y_{\alpha}} G_{r}(t, y), \quad \frac{\partial G_{r+1}}{\partial t}=G_{r}
$$

where

$$
G_{0}(t, y)=-\frac{1}{\sqrt{2} \pi} \int_{t>\psi} \frac{\mathrm{d} w}{\sqrt{t-\psi(w, y)}}
$$

for $\mu=1$ and

$$
G_{0}(t, y)=-\sqrt{\left|\operatorname{det} \psi_{w w}\right|} \frac{1}{2 \pi} \iint_{-\infty}^{\infty} \delta(t-\psi(w, y)) \mathrm{d} w_{1} \mathrm{~d} w_{2}
$$

for $\mu=2$.

The integral in equation (22a) can be expressed in terms of elementary functions and complete elliptic integrals if the degree of $\psi$ does not exceed 4 . In the second case we apply the following lemma:

LEMMA. The integral in equation (22b) can be expressed in terms of elementary functions and elliptic integrals if the following conditions are satisfied:

(i) $\psi=a w_{1}^{2}+b w_{1}+c$;

(ii) $b^{2}-4 a c$ is a polynomial in $w_{2}$ of degree $\leq 4$.

Proof. It is easy to check that

$$
\int_{y}^{\infty} \delta\left(x^{2}-z\right) \mathrm{d} x=\frac{1}{2}\left\{\begin{aligned}
H\left(z-y^{2}\right) z_{+}^{-1 / 2} & \text { if } \quad y>0 \\
{\left[1+H\left(y^{2}-z\right)\right] z_{+}^{-1 / 2} } & \text { if } \quad y<0 .
\end{aligned}\right.
$$


In view of (i) $\psi=a\left(w_{1}+b / 2 a\right)^{2}+c-b^{2} / 4 a$ and Eq. ( $\left.\sharp\right)$ can be applied with $z=c-b^{2} / 4 a$, whence the assertion follows from (ii).

It will be seen in Sections 4 and 6 that several canonical time-domain functions satisfy the above conditions. In such cases very efficient algorithms can be developed for numerical evaluation of $V(x, t)$.

4. Time-domain canonical solutions at caustics. Stable caustic singularities fall into two categories:

(i) cuspoid caustics,

(ii) umbilics.

In a 3-dimensional space $\mathcal{X} \subset \mathbb{R}^{3}$ the former are generated by one-dimensional focusing of ray tubes while the latter involve focusing of a ray tube to a point.

Canonical functions $G_{0}$ corresponding to cuspoid caustics are derived by substituting the universal unfoldings of the singularities $A_{k}, k \geq 3$ in Eq. (22a):

$$
\psi=\frac{w^{k+1}}{k+1}+\sum_{l=0}^{k-1} y_{k} w^{k}
$$

$[1,22]$. The asymptotic contribution of an $A_{k}$-singularity is given by equation (22a). For $k=2,3$ it can be expressed in terms of complete elliptic integrals. Detailed expressions can be found in [15].

Canonical expressions for umbilic singularities are obtained by substituting appropriate polynomials in two variables in (22b). In particular the lemma from the preceding section applies to the elliptic and hyperbolic umbilic $D_{4}^{\mp}$ :

$$
\psi=w_{1}^{2} w_{2} \mp w_{2}^{3}+y_{3} w_{1}^{2}+y_{1} w_{1}+y_{2} w_{2}+y_{0} .
$$

5. Singularities at shadow boundaries and at intersections of shadow boundaries. We consider the case of a principal ray field bounded by one or several shadow boundaries. In accordance with the GTD [18] the asymptotics of the wave field can be expressed in terms of the principal ray field and the diffracted ray fields associated with the shadow boundaries.

A shadow boundary is the projection of the intersection of two Lagrangian submanifolds $\mathcal{I}=\Lambda_{1} \cap \Lambda_{2}$. The set $\mathcal{I}$ is an isotropic subvariety of $T^{*} \mathcal{X}: \omega \mid \mathcal{I}=0$. The following theorem describes generating families of functions of isotropic subvarieties [16]:

THEOREM 3. An isotropic subvariety $\mathcal{I} \subset T^{*} \mathcal{X}$ can be locally represented in terms of a holomorphic generating family

$$
\begin{gathered}
\phi: \tilde{\mathcal{U}} \times \tilde{\mathcal{V}} \times \mathcal{X} \rightarrow \mathbb{R} \\
\tilde{\mathcal{U}} \subset \mathbb{C}^{M_{1}}, \tilde{\mathcal{V}} \subset \mathbb{C}^{M_{2}}, \mathcal{X} \subset \mathbb{C}^{n}
\end{gathered}
$$

by the equation

(25) $\mathcal{I}=\left\{(x, p) \in T^{*} \mathcal{X} \mid \exists \tilde{u} \in \tilde{\mathcal{U}}: p=\frac{\partial \phi(\tilde{u}, 0, x)}{\partial x}, \frac{\partial \phi(\tilde{u}, 0, x)}{\partial \tilde{u}}=0, \frac{\partial \phi(\tilde{u}, 0, x)}{\partial \tilde{v}}=0\right\}$. 
Remark. $\mathcal{I}$ is a submanifold of $T^{*} \mathcal{X}$ if the 1 -forms

$$
\mathrm{d} \frac{\partial \phi}{\partial \tilde{u}_{i}}, i=1, \ldots, M_{1} ; \mathrm{d} \frac{\partial \phi}{\partial \tilde{v}_{j}}, j=1, \ldots, M_{2}
$$

are linearly independent of

$$
\mathcal{M}=\left\{(\tilde{u}, \tilde{v}, x) \in \tilde{\mathcal{U}} \times \tilde{\mathcal{V}} \times \mathcal{X} \mid \tilde{v}=0, \frac{\partial \phi}{\partial \tilde{u}}=0, \frac{\partial \phi}{\partial \tilde{v}}=0\right\}
$$

$\mathcal{I}$ is the intersection of the following Lagrangian submanifolds:

$$
\begin{aligned}
\Lambda_{P}= & \left\{(x, p) \in T^{*} \mathcal{X} \mid \exists(\tilde{u}, \tilde{v}) \in \tilde{\mathcal{U}} \times \tilde{\mathcal{V}}:\right. \\
& \left.p=\frac{\partial \phi(\tilde{u}, \tilde{v}, x)}{\partial x}, \frac{\partial \phi(\tilde{u}, \tilde{v}, x)}{\partial \tilde{u}}=0, \frac{\partial \phi(\tilde{u}, \tilde{v}, x)}{\partial \tilde{v}}=0\right\}
\end{aligned}
$$

and

$$
\begin{aligned}
\Lambda_{D_{k}}=\left\{(x, p) \in T^{*} \mathcal{X} \mid \exists(\tilde{u}, \tilde{v}) \in \tilde{\mathcal{U}} \times \tilde{\mathcal{V}}: \tilde{v}_{k}=0, p=\frac{\partial \phi(\tilde{u}, \tilde{v}, x)}{\partial x},\right. \\
\frac{\partial \phi(\tilde{u}, \tilde{v}, x)}{\partial \tilde{u}}=0, \frac{\partial \phi\left(\tilde{u}, \tilde{v}_{1}, \ldots, \tilde{v}_{k-1}, 0, \tilde{v}_{k+1}, \ldots, \tilde{v}_{M_{2}}\right)}{\partial \tilde{v}_{l}}=0 \\
\left.\quad \text { for } \quad l=1, \ldots, M_{2}, l \neq k\right\}
\end{aligned}
$$

for $k=1, \ldots, M_{2}$. This observation suggests the following ansatz for asymptotic solutions at shadow boundaries:

$$
U(x, \lambda) \sim \int_{\hat{\mathcal{U}} \times \hat{\mathcal{V}}_{+}} \mathrm{d} \tilde{u} \mathrm{~d} \tilde{v} a(\tilde{u}, \tilde{v}, x, \lambda) \exp [\mathrm{i} \lambda \phi(\tilde{u}, \tilde{v}, x)]
$$

where $\hat{\mathcal{U}}:=\tilde{\mathcal{U}} \cap \mathbb{R}^{M_{1}}, \hat{\mathcal{V}}_{+}:=\left\{\tilde{v} \in \tilde{\mathcal{V}} \cap \mathbb{R}^{M_{2}} \mid \tilde{v}_{l} \geq 0 \quad\right.$ for $\left.\quad l=1, \ldots, M_{2}\right\}$. The interior stationary points of $(29)$ correspond to the subset

$$
\begin{aligned}
\Lambda_{P}^{+}:= & \left\{(x, p) \in T^{*} \mathcal{X} \mid \exists(\tilde{u}, \tilde{v}) \in \hat{\mathcal{U}} \times \hat{\mathcal{V}}_{+}:\right. \\
& \left.p=\frac{\partial \phi(\tilde{u}, \tilde{v}, x)}{\partial x}, \frac{\partial \phi(\tilde{u}, \tilde{v}, x)}{\partial \tilde{u}}=0, \frac{\partial \phi(\tilde{u}, \tilde{v}, x)}{\partial \tilde{v}}=0\right\}
\end{aligned}
$$

of $\Lambda_{P}$ while the points of $\bigcup_{k} \Lambda_{D_{k}} \backslash \Lambda_{P}$ correspond to the boundary stationary points of the second kind of (29).

After some modifications Theorem 2 also applies to equation (29). Indeed, assuming that for $x \in \Omega \subset \mathcal{X}$ all the interior stationary points lie in a subset $\mathcal{D} \subset \tilde{\mathcal{U}} \times \tilde{\mathcal{V}}_{+}$which does not intersect $\partial\left(\tilde{\mathcal{U}} \times \tilde{\mathcal{V}}_{+}\right)$, we can apply the partition of unity argument to split the contributions of interior stationary points from the contributions of the boundary

$$
U=U_{\mathcal{D}}+U_{B d}+U_{R}
$$

where

$$
U_{R}=\mathrm{O}\left[\lambda^{-\infty}\right], P\left(x, D_{x}, \lambda\right) U_{R}=\mathrm{O}\left[\lambda^{-\infty}\right]
$$

and $U_{B d}=\sum_{k=1}^{M_{2}} U_{k}$, where

(31) $U_{k}=\frac{1}{\mathrm{i} \lambda} \int_{\tilde{\mathcal{V}}_{+} \cap\left\{\tilde{v}_{k}=0\right\}} \mathrm{d} \tilde{v}_{1} \ldots \widehat{\mathrm{d} \tilde{v}_{k}} \ldots \mathrm{d} \tilde{v}_{M_{2}} \int_{\tilde{\mathcal{U}}} \mathrm{d} \tilde{u} a(\tilde{u}, \tilde{v}, x, \lambda) \frac{\phi_{\tilde{v}_{k}}}{\phi_{\tilde{u}}^{2}+\phi_{\tilde{v}}^{2}} \exp [\mathrm{i} \lambda \phi(\tilde{u}, \tilde{v}, x)]$ 
and $\phi_{\tilde{u}}^{2}:=\sum_{l} \phi_{\tilde{u}_{l}}^{2}, \phi_{\tilde{v}}^{2}:=\sum_{l} \phi_{\tilde{v}_{l}}^{2}$. In order to derive equations (30) and (31) it is enough to substitute $R \mathrm{e}^{\mathrm{i} \lambda \phi}$, with $R:=(\mathrm{i} \lambda)^{-1}\left\langle\phi_{z} \mid \phi_{z}\right\rangle^{-1}\left\langle\phi_{z} \mid \frac{\partial}{\partial z}\right\rangle, z=(\tilde{u}, \tilde{v})$, for $\mathrm{e}^{\mathrm{i} \lambda \phi}$ and repeatedly integrate by parts. The operator $R$ is well-defined outside $\mathcal{D}$.

If we assume that the boundary stationary points of $\phi$ are isolated the diffracted wave field $U_{B d}$ can be expanded in a ray series

$$
U_{B d} \sim \sum_{k, \sigma}(\mathrm{i} \lambda)^{-k} B_{k, \sigma}(x) \mathrm{e}^{\mathrm{i} \lambda S^{(\sigma)}(x)}
$$

with

$$
B_{0, \sigma}(x)=\left.a_{0}(\tilde{u}, \tilde{v}, x)\left|\operatorname{det} \phi_{w w}\right|^{-1 / 2} \phi_{\tilde{v}_{l}}^{-1}\right|_{\tilde{v}_{l}=0, \phi_{w}=0}
$$

for some $l \leq M_{2}$ and $w:=\left(\tilde{u}, \tilde{v}_{1}, \ldots, \widehat{\tilde{v}_{l}}, \ldots, \tilde{v}_{M_{2}}\right)$. Theorem 2 can now be applied to both $U_{\mathcal{D}}$ and $U_{B d}$.

The denominator of $B_{0, \sigma}(x)$, Eq. (33), involves two expressions that vanish at symplectic singularities:

(1) $\phi_{\tilde{v}_{l}}=0$ at the shadow boundary $\pi\left(\Lambda_{D_{l}} \cap \Lambda_{P}\right)$;

(2) $\operatorname{det} \phi_{w w}=0$ at the diffracted-wave caustics.

The singularity of $B_{0, \sigma}(x)$ associated with the vanishing of $\phi_{\tilde{v}_{l}}$ corresponds to the singularity of $D / r_{1}^{1 / 2}$ in the GTD formula for the amplitude of an edge-diffracted signal [18]: $B_{0}=D c^{1 / 2}|\lambda|^{-1 / 2}\left(r_{2}^{0} / r_{1} r_{2}\right)^{1 / 2}$, where $D$ denotes the diffraction coefficient, $r_{1}, r_{2}$ denote the principal radii of the diffracted wavefront, $r_{1}^{0}=0, r_{2}^{0}$ denote their values at the diffraction point on the same ray and $c(x)$ denotes the propagation speed [13, 14].

The same method allows computation of the contribution of a corner point for $M_{2}=2$. For simplicity we restrict our attention to the special case

$$
U(x, \lambda)=\int_{\tilde{v}_{1}, \tilde{v}_{2} \geq 0} a \mathrm{e}^{\mathrm{i} \lambda \phi} \mathrm{d} \tilde{v}_{1} \mathrm{~d} \tilde{v}_{2} .
$$

In this case

$$
U_{B d}=-\frac{1}{\mathrm{i} \lambda}\left[\left.\int_{0}^{\infty} \mathrm{d} v_{2} \frac{\phi_{\tilde{v}_{1}}}{\phi_{\tilde{v}}^{2}} a \mathrm{e}^{\mathrm{i} \lambda \phi}\right|_{\tilde{v}_{1}=0}+\left.\int_{0}^{\infty} \mathrm{d} \tilde{v}_{1} \frac{\phi_{\tilde{v}_{2}}}{\phi_{\tilde{v}}^{2}} a \mathrm{e}^{\mathrm{i} \lambda \phi}\right|_{\tilde{v}_{2}=0}\right]+\text { h.o.t. }
$$

where $\phi_{\tilde{v}}^{2}:=\phi_{\tilde{v}_{1}}^{2}+\phi_{\tilde{v}_{2}}^{2}$. Substituting $\left(\mathrm{i} \lambda \phi_{\tilde{v}_{k}}\right)^{-1} \frac{\partial \mathrm{e}^{\mathrm{i} \lambda \phi}}{\partial \tilde{v}_{k}}$ for $\mathrm{e}^{\mathrm{i} \lambda \phi}$ in the integral over $\tilde{v}_{k}$, $k=1,2$, and integrating by parts we get the lowest-order asymptotic contribution of the corner point in the form

$$
\frac{1}{(\mathrm{i} \lambda)^{2}} a\left[\frac{\phi_{\tilde{v}_{1}}}{\phi_{\tilde{v}_{2}}}+\frac{\phi_{\tilde{v}_{2}}}{\phi_{\tilde{v}_{1}}}\right] \mathrm{e}^{\mathrm{i} \lambda \phi} /\left.\phi_{\tilde{v}}^{2}\right|_{\tilde{v}_{1}=\tilde{v}_{2}=0} .
$$

The following coalescences are possible in addition to the coalescences of interior and boundary stationary points:

(i) an interior stationary point and two boundary stationary points coalesce with the corner $(0,0)$;

(ii) one boundary stationary point coalesces with the corner. 
In case (i) both $\phi_{\tilde{v}_{1}}$ and $\phi_{\tilde{v}_{2}}$ tend to zero and the behaviour of expression (36) is quite complex. In case (ii) one of these derivatives tends to zero while the other remains different from zero. Case (i) corresponds to the intersection of two shadow boundaries of the principal signal (which also lies on the shadow boundaries of the edge-diffracted signals) while case (ii) occurs at the shadow boundary of an edge diffracted signal.

6. Canonical functions for shadow boundary singularities. We now consider the asymptotic contribution of a boundary or corner singularity $[1,23]$ of the phase function. Applying the version of the Splitting Lemma presented in [23] we get

$$
\phi(\tilde{u}, \tilde{v}, x)=\psi\left(u_{1}, \ldots, u_{m_{1}}, v_{1}, \ldots, v_{m_{2}}, F(x)\right)+\sum_{k=m_{1}+1}^{M_{1}} \frac{1}{2} \sigma_{k} u_{k}^{2}+\sum_{l=m_{2}+1}^{M_{2}} \rho_{l} v_{l}
$$

where $\sigma_{k}, \rho_{l} \in\{-1,1\}$,

$$
\psi(u, v, y)=\psi_{0}(u, v)+\sum_{j=1}^{\mu} y_{j} h_{j}(u, v)+y_{0}
$$

is a universal unfolding of a boundary or corner singularity $\psi_{0}$ of boundary corank $m_{2}>0$. The monomials $\left\{h_{j}, j=1, \ldots, \mu\right\}$ constitute a basis of

$$
\mathcal{M} /\left\langle\partial \psi_{0} / \partial u_{1}, \ldots, \partial \psi_{0} / \partial u_{m_{1}}, v_{1} \partial \psi_{0} / \partial v_{1}, \ldots, v_{m_{2}} \partial \psi_{0} / \partial v_{m_{2}}\right\rangle
$$

where $\mathcal{M}$ denotes the maximal ideal of $\mathcal{C}_{0}^{\infty}(\hat{\mathcal{U}} \times \hat{\mathcal{V}})$. Standard asymptotic methods [7] can be applied to the integrals over the inessential variables $u_{k}, k>m_{1}$, and $v_{l}, l>m_{2}$, yielding a factor

$$
N=\left(\frac{2 \pi}{|\lambda|}\right)^{\left(M_{1}-m_{1}\right) / 2} \exp \left(\mathrm{i} \frac{\pi}{4} \sum_{k=m_{1}+1}^{M_{1}} \operatorname{sgn} \lambda\right) \prod_{l=m_{2}+1}^{M_{2}} \frac{1}{\mathrm{i} \lambda \rho_{l}}
$$

which is irrelevant in the following and will be disregarded.

The contribution of the singularity $\psi_{0}$ to the wave field $U(x, \lambda)$ assumes the form

$$
I=\sum_{r=0}^{\infty} \frac{1}{(\mathrm{i} \lambda)^{r}} I_{r}, \quad I_{r}=\int_{\mathcal{U} \times \mathcal{V}_{+}} b_{r}(u, v, x) \mathrm{e}^{\mathrm{i} \lambda \psi(u, v, F(x))} \mathrm{d} u \mathrm{~d} v
$$

with $\mathcal{U} \subset \mathbb{R}^{m_{1}}, \mathcal{V}_{+} \subset \mathbb{R}^{m_{2}}, b_{r}:=a_{r} e_{k} \partial(\tilde{u}, \tilde{v}) / \partial(u, v)$.

The amplitude function $b_{r}$ can now be expanded in the basis $\left\{h_{j}\right\}$ modulo $\partial \psi / \partial u_{1}, \ldots, \partial \psi / \partial u_{m_{1}}, v_{1} \partial \psi / \partial v_{1}, \ldots, v_{m_{2}} \partial \psi / \partial v_{m_{2}}$. By the generalized Malgrange preparation theorem [8]

$$
\begin{aligned}
b_{r}(u, v, x)=c_{r 0}(x) & +\sum_{j=1}^{\mu} c_{r j}(x) h_{j}(u, v)+\left.\sum_{k=1}^{m_{1}} f_{k}(u, v, x) \frac{\partial \psi}{\partial u_{k}}\right|_{y=F(x)} \\
& +\left.\sum_{l=1}^{m_{2}} v_{l} g_{l}(u, v, x) \frac{\partial \psi}{\partial v_{l}}\right|_{y=F(x)} .
\end{aligned}
$$

Integration by parts shows that the last two terms of Eq. (40) give a contribution of order $\mathrm{O}\left[(\mathrm{i} \lambda)^{-r-1}\right]$ to $I$ and consequently can be absorbed in $I_{r+1}$. Eq. (40) can be 
rewritten in the form

$$
I_{r}=\left.\frac{1}{\mathrm{i} \lambda} \sum_{j=0}^{\mu} c_{r j}(x) \frac{\partial}{\partial y_{j}} \int_{\mathcal{U} \times \mathcal{V}_{+}} \mathrm{e}^{\mathrm{i} \lambda \psi} \mathrm{d} u \mathrm{~d} v\right|_{y=F(x)} .
$$

The asymptotic contribution $F(x, t)$ of the singularity to the time-domain wave field $V(x, t)$, obtained by substitution of (40)-(41) in (13) with $m:=m_{1}+m_{2}$, is given by the asymptotic expansion (21) with the canonical functions

$$
G_{0}(t, y)=-\frac{1}{\sqrt{2} \pi} \int_{\substack{w>0 \\ t>\psi}} \frac{\mathrm{d} w}{\sqrt{t-\psi(w, y)}}
$$

for $m_{1}=0, m_{2}=1$,

$$
G_{0}(t, y)=-\frac{\sqrt{\left|\operatorname{det} D^{2} \psi(0, y)\right|}}{2 \pi} \int_{-\infty}^{\infty} \int_{0}^{\infty} \delta(t-\psi(u, v, y)) \mathrm{d} u \mathrm{~d} v
$$

for $m_{1}=m_{2}=1$,

$$
G_{0}(t, y)=-\frac{\sqrt{\left|\operatorname{det} D^{2} \psi(0, y)\right|}}{2 \pi} \int_{0}^{\infty} \int_{0}^{\infty} \delta(t-\psi(u, v, y)) \mathrm{d} u \mathrm{~d} v
$$

for $m_{1}=0, m_{2}=2 . D^{2} \psi$ denotes the Hessian of $\psi$ with respect to $(u, v)$.

For the single shadow boundary we apply the universal unfoldings of the boundary singularities listed in [1]. The following singularities have codimension $\leq 4$ :

$$
B_{k}: m_{1}=0, m_{2}=1, \psi(v, y)=\frac{v^{k}}{k}+\sum_{l=0}^{k-1} y_{l} v^{l}
$$

for $k \geq 2$,

$$
C_{k}: m_{1}=m_{2}=1, \psi(u, v, y)=\left(u-y_{k-1}\right) v+\frac{u^{k}}{k}+\sum_{l=0}^{k-2} y_{l} u^{l}
$$

for $k \geq 3$,

$$
F_{4}: m_{1}=m_{2}=1, \psi(u, v, y)=u^{3} \pm v^{2}+y_{3} u v+y_{1} u+y_{2} v+y_{0}
$$

with $v \geq 0$.

The $B_{2}$ singularity represents a shadow boundary $[10,12]$. The $B_{k}$ series for $k>2$ involves caustics of the principal signal tangent to the shadow boundary. They break off at the shadow boundary and are called "broken caustics" in [19]. The $C_{k}$ series involves caustics of the diffracted waves (called the penumbra caustics in [19]).

In the case of the $F_{4}$ singularity the principal-wave and diffracted-wave caustics intersect at a common focus of the two kinds of waves. The principal wavefront breaks off at its intersection $\mathcal{E}$ with the shadow boundary. The principal rays through $\mathcal{E}$ are simultaneously diffracted rays. In the isotropic case (the rays are orthogonal to the corresponding wavefronts) the diffracted and principal rays have a common focus if a center of curvature of the principal wavefront coincides with a center of curvature of $\mathcal{E}$, i.e. if $\mathcal{E}$ is tangent to a principal curvature direction of the principal wavefront. 
The canonical function for $B_{k}$ are given by Eq. (42a). For $B_{2}$ Eq. (42a) can be expressed in terms of arc sine, while for $B_{3}, B_{4}$ it involves incomplete elliptic functions.

The canonical functions of the $C_{k}$ series are distributional limits of algebraic functions defined in the lower half of the complex $t$-plane. The leading term of the asymptotic expansion (14) for $C_{k}$ is

$$
F_{0}(x, t)=2 \Re \int_{0}^{\infty} \mathrm{d} \lambda \frac{\mathrm{i} \lambda}{4 \pi^{2}} \int_{-\infty}^{\infty} \mathrm{d} u \int_{0}^{\infty} \mathrm{d} v \mathrm{e}^{\mathrm{i} \lambda\left[\left(u-y_{k-1}\right) v+\zeta-t\right]}
$$

where

$$
\zeta=\frac{u^{k}}{k}+\sum_{l=0}^{k-2} y_{l} u^{l} .
$$

Integration over $v$ can be carried out first

$$
\int_{0}^{\infty} \mathrm{d} v \mathrm{e}^{\mathrm{i} \lambda\left[\left(u-y_{k-1}\right) v+\zeta\right]}=\frac{\mathrm{i}}{\lambda} \frac{1}{u-y_{k-1}+\mathrm{i} 0} \mathrm{e}^{\mathrm{i} \lambda(\zeta-t)}
$$

leading to

$$
G_{0}(t, y)=\frac{1}{2 \pi^{2}} \Re \int_{-\infty}^{\infty} \frac{1}{u-y_{k-1}+\mathrm{i} 0} \frac{1}{t-\zeta-\mathrm{i} 0} \mathrm{~d} u
$$

The asymptotic contribution associated with $\Lambda_{P}$ is now represented by the residue while the saddle points represent the diffracted waves. The canonical function (49) can be evaluated in terms of algebraic functions by closing the contour in the complex $u$-plane and applying the residue calculus $[10,12]$.

For the intersection of two shadow boundaries we apply the universal unfoldings of the singularities listed in [23]. The first item in the list of singularities of functions in a quarter plane $\mathbb{R}_{+}^{2}$ has the following form:

$$
\begin{aligned}
\psi(v, M, y) & =y_{0}+y_{1} v_{1}+y_{2} v_{2}+M v_{1} v_{2}+\iota_{1} v_{1}^{2}+\iota_{2} v_{2}^{2}, \\
\iota_{k} & \in\{+1,-1\} \quad \text { for } k=1,2 ; \quad M \neq \pm 2 .
\end{aligned}
$$

It involves two kinds of parameters: the unfolding parameters $y_{l}$ and a module $M$. The transformation of the phase function $\phi$ to the polynomial $\psi$ assumes the following form:

$$
\phi(u, x)=\psi(f(u, x), M, g(x))
$$

with a constant $M$. In the case of diffraction by a sector and a convex principal wavefront $M=-2 \cos \theta$, where $\theta$ is the angle at the vertex [14]. The canonical time-domain function assumes the form

$$
G_{0}(t, y)=-\frac{\sqrt{|\Delta|}}{2 \pi} \int_{0}^{\infty} \int_{0}^{\infty} \mathrm{d} v_{1} \mathrm{~d} v_{2} \delta(t-\psi(v, M, y))
$$

with $\Delta=4 \iota_{1} \iota_{2}-M^{2}$, is expressible in terms of the arc tangent function [14].

Subsequent items on Siersma's list of corner singularities allow for cusped sectors as well as for intersecting shadow boundaries tangent to a caustic at their intersection. Explicit time-domain solutions can be derived for the lowest codimensions. 
7. Concluding remarks. The eikonal function associated with a singularity $A_{k}, D_{k}$, $B_{k}, C_{k}, F_{4}$ of codimension $\mu$ has $\mu+1$ branches. The $\mu+1$ values of the eikonal at a point are given by a set of polynomial functions of the $\mu+1$ unfolding parameters. The values of the eikonal at a point $x$ can be determined by two-point ray tracing and the unfolding parameters can subsequently be determined by solving an algebraic system of equations $[10,12,13]$. For each $r$ the parameters $c_{r j}(x)$ can be calculated from the ray amplitudes of order $r$ by solving a linear system of equations.

The canonical function (52) involves one principal wavefront, two edge-diffracted wavefronts and a vertex-diffracted wavefront. The vertex-diffracted wavefront is generated by the asymptotic contribution of the corner $(0,0)$. The 3 unfolding parameters as well as the module $M$ can be determined from the 4 eikonals.

Acknowledgement. I am indebted to an anonymous reviewer for reminding me of Duistermaat's paper [6].

\section{References}

[1] V. I. Arnol' d, A. N. Varchenko and S. M. Husein-Zade, Singularities of Differentiable Maps, Vol. I, Birkhäuser Verlag, Basel, 1985.

[2] S. Benenti and W. M. Tulczyjew, The Geometrical Meaning and Globalization of the Hamilton-Jacobi Method, in: Differential geometrical methods in mathematical physics, Proc. Conf. Aix-en-Provence//Salamanca 1979, Lecture Notes in Math. 836, Springer, Berlin, 1980, 9-21.

[3] M. G. Brown and F. D. Tappert, Causality, caustics and the structure of transient wavefields, J. Acoust. Soc. Amer. 85 (1986), 251-255.

[4] R. Burridge, The reflection of a pulse in a solid sphere, Proc. Roy. Soc. London A276 (1962), 367-400.

[5] J. N. L. Connor, A method for the numerical evaluation of the oscillatory integrals associated with cuspoid catastrophes: applications to Pearcey's integral and its derivatives, J. Phys. A 15 (1982), 1179-1190.

[6] J. J. Duistermaat, Oscillatory integrals, Lagrange immersions and unfolding of singularities, Comm. Pure Appl. Math. 27 (1974), 207-281.

[7] M. V. Fedoryuk, Saddle Point Method, Nauka, Moscow, 1977 (in Russian).

[8] M. Golubitsky and V. Guillemin, Stable Mappings and their Singularities, SpringerVerlag, New York, 1973.

[9] V. Guillemin and S. Sternberg, Geometric Asymptotics, American Mathematical Society, Providence, RI, 1977.

[10] A. Hanyga, Boundary effects in Asymptotic Diffraction Theory, Part I-III, Seismo-series (int. reports ISEP Univ. of Bergen), Vol. 35-37, Bergen, 1989.

[11] A. Hanyga, Local behaviour of wavefields at simple caustics, Seismo-series (int. reports ISEP Univ. of Bergen), Vol. 38, 1989.

[12] A. Hanyga, Numerical applications of Asymptotic Diffraction Theory, in: Mathematical and Numerical Aspects of Wave Propagation, Univ. of Delaware, June 1993, R. Kleinman et al. (eds.), SIAM, Philadelphia, 1993. 
[13] A. Hanyga, Asymptotic Diffraction Theory applied to edge-vertex diffraction, in: Proc. 7th Conference on Waves and Stability in Continuous Media, Bologna, Nov. 1993, S. Rionero \& T. Ruggeri (eds.), World Scientific Publishing, Singapore, 1994.

[14] A. Hanyga, Asymptotic vertex and edge diffraction, Geophys. J. Int. 122 (1995), 277-290.

[15] A. Hanyga and M. Seredy/nska, Diffraction of pulses in the vicinity of simple caustics and caustic cusps, Wave Motion 14 (1991), 101-121.

[16] S. Janeczko, On isotropic submanifolds and evolution of quasi-caustics, Pacific J. Math. 158 (1993), 317-334.

[17] S. Janeczko and G. Plotnikova, Sur la structure de quasi-caustiques en diffraction géometrique, C. R. Acad. Sci. Paris Sér. I Math. 311 (1990), 543-546.

[18] J. B. Keller, Geometrical Theory of Diffraction, J. Opt. Soc. Amer. 52 (1966), 116-130.

[19] Yu. A. Kravtsov and Yu. I. Orlov, Caustics, Catastrophes and Wave Fields, SpringerVerlag, Berlin, 1993.

[20] V. P. Maslov and V. M. Fedoryuk, Semi-classical approximation in quantum mechanics, D. Reidel, Doordrecht, 1981.

[21] F. Pham, Singularités des systèmes differentiels de Gauss-Manin, Progr. Math. 2, Birkhäuser, Basel, 1979.

[22] T. Poston and I. Stewart, Catastrophe Theory and Its Applications, Pitman, London, 1978.

[23] D. Siersma, Singularities of functions on boundaries, corners etc., Quart. J. Math. Oxford (2) 32 (1981), 119-127.

[24] D. Stickler, D. S. A hluwalia and L. Ting, Application of Ludwig's uniform progressing wave ansatz to a smooth caustic, J. Acoust. Soc. Amer. 69 (1981), 1673-1681.

[25] A. Weinstein, Lectures on Symplectic Manifolds, in: CBMS Conference Series, American Mathematical Society, Vol. 29, Providence, RI, 1977. 